\title{
Submitted: 7 Comparison of sonographic hepatorenal ratio Accepted: 13.07.2020 \\ Published: and the degree of hepatic steatosis in magnetic resonance imaging-proton density fat fraction
}

28.09.2020

\section{Keywords}

HR ratio, MRI-PDFF, hepatic steatosis

\author{
Natthaporn Tanpowpong, Sineenart Panichyawat
}

\begin{abstract}
Diagnostic Radiology, Faculty of Medicine, Chulalongkorn University, Bangkok, Thailand Correspondence: Sineenart Panichyawat, MD, Division of Diagnostic Radiology, Department of Radiology, Faculty of Medicine, Chulalongkorn University, King Chulalongkorn Memorial Hospital, Bangkok, Thailand; e-mail: B_am3z@hotmail.com
\end{abstract}

DOI: $10.15557 / J \circ U .2020 .0028$

\begin{abstract}
Objectives: Conventional ultrasonography can provide only semi-quantitative assessment of hepatic steatosis. The aim of this study was to assess sonographic hepatorenal ratio to quantify the severity of fatty liver. Methods: We performed a retrospective analysis of 179 patients with various liver diseases who underwent abdominal magnetic resonance imaging and ultrasonography on the same day. The hepatorenal ratio was calculated by the ratio between the mean echo intensity in regions of interests of the liver and regions of interests of the right renal cortex. Magnetic resonance imaging-proton density fat fraction was used as standard reference for steatosis grading. The effect of fibrosis measured by magnetic resonance elastography on the degree of correlation was also assessed. Results: The hepatorenal ratio was highly correlated with magnetic resonance imaging-proton density fat fraction (Spearman's coefficient $=0.83)(p<0.001)$. High correlation of hepatorenal ratio with magnetic resonance imaging-proton density fat fraction was observed in patients with less than stage 2 fibrosis $(p<0.001)$, whereas moderate correlation of hepatorenal ratio with magnetic resonance imaging-proton density fat fraction was found in patients with $\geq$ stage 2 fibrosis or higher $(p<0.001)$. The hepatorenal ratio cutoff point for prediction of grade 1 hepatic steatosis was 1.18 with sensitivity of $90.0 \%$ and specificity of $80.0 \%$. The hepatorenal ratio cutoff point for prediction of grade 2 and grade 3 hepatic steatosis was 1.55 and 1.60, respectively, with sensitivity greater than $90 \%$ and specificity greater than $80 \%$. Conclusions: The hepatorenal ratio could become an effective quantitative tool for hepatic steatosis alternative to magnetic resonance imaging-proton density fat fraction. Application should be careful in the group of patients with stage 2 liver fibrosis or higher.
\end{abstract}

\section{Introduction}

Hepatic steatosis is a condition of excessive intrahepatocytic accumulation of fat. Steatosis is the hallmark of non-alcoholic fatty liver disease (NAFLD) and also occurs in patients with alcohol abuse, viral hepatitis, HIV and those receiving certain pharmacological treatment ${ }^{(1,2)}$. NAFLD is the most common chronic liver disease affecting approximately $30 \%$ of the general population ${ }^{(2,3)}$. Steatosis can progress to liver fibrosis and, eventually, cirrhosis, and it can also worsen the course of disease in patients with viral hepatitis ${ }^{(4,5)}$.
A quantitative tool for assessment and monitoring of hepatic steatosis is needed in clinical practice. Liver biopsy still remains the gold standard for the assessment of liver $\mathrm{fat}^{\left({ }^{(6)}\right.}$. However, its routine clinical use is limited due to its invasiveness with a small potential risk of life-threatening complications, sampling errors and inter-observer variability $^{(7-9)}$.

Noninvasive imaging techniques, including MR spectroscopy $\left({ }^{1} \mathrm{H}\right.$-MRS) and magnetic resonance imaging-proton density fat fraction (MRI-PDFF), can accurately measure 


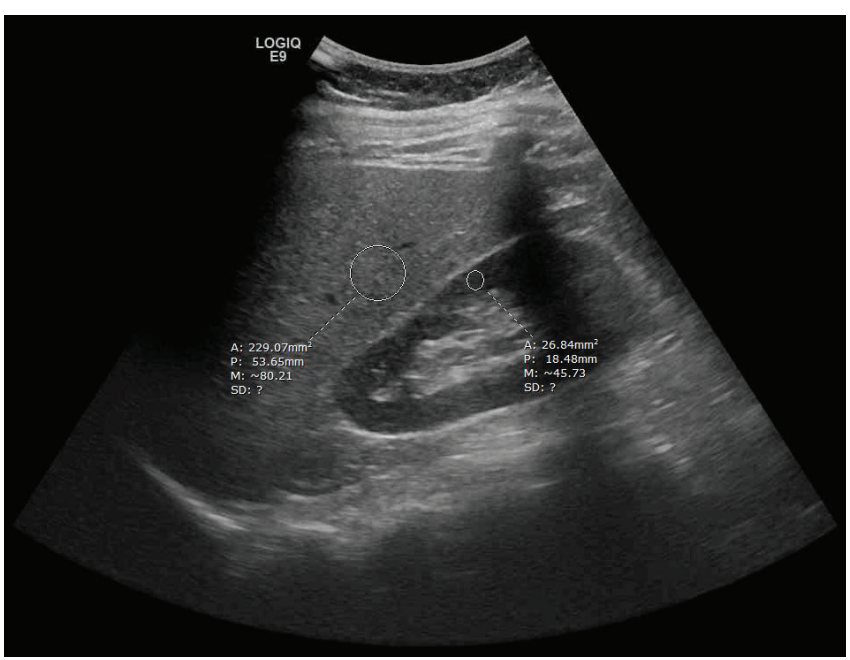

Fig. 1. The hepatic and right renal ROIs were selected at the same depth along the focusing area near the center of the image to avoid image distortion effects and beam attenuation

hepatic fat. ${ }^{1} \mathrm{H}$ MRS is accepted as the noninvasive reference standard for liver fat quantification ${ }^{(10)}$.

PDFF measured by MRI is a direct measure of the fraction of mobile triglyceride protons relative to those in water $^{(10)}$. MRI-PDFF proved to have excellent agreement with ${ }^{1} \mathrm{H}-\mathrm{MRS}^{(11-15)}$ and to be closely correlated with liver histology ${ }^{(14,16,17)}$, as well as has been recently considered as a standardized MR biomarker of steatosis used in clinical practice and research. Despite the accuracy of the MR techniques, their use is restricted due to high operational cost and considerable time consumption.

Ultrasonography (US) is often the first imaging modality used to detect hepatic steatosis. However, conventional ultrasonography can provide only qualitative or semiquantitative assessment based on liver echogenicity, vessel visibility, and beam attenuation. Overall sensitivity and specificity of ultrasonography previously reported were about $60-94 \%$ and $66-97 \%$, respectively ${ }^{(18)}$. Prior studies have investigated sonographic parameters in an attempt to overcome subjectivity and qualitative limitations, such as hepatic-renal differences ${ }^{(19)}$ and hepatorenal (HR) ratio ${ }^{(20-25)}$. The diagnostic value of the HR ratio varies among studies depending on different standard references and study populations; therefore further validation for clinical application is still needed. Moreover, a direct comparison between sonographic HR ratio and MRI-PDFF has not been performed yet.

The aim of this study was to evaluate the sonographic HR ratio for the diagnosis and quantification of hepatic steatosis using MRI-PDFF as reference.

\section{Materials and methods}

The study was approved by the Institutional Review Board. No informed consent was required due to the retrospective nature of the study.

\section{Subjects}

Patients aged $\geq 18$ years with various liver diseases who had underwent abdominal MR imaging and ultrasonography on the same day at King Chulalongkorn Memorial Hospital (KCMH) between January 2016 and December 2017 were assessed retrospectively. All images were reviewed using Picture Archiving and Communication System (PACS; Fujifilm Synapse, Tokyo, Japan).

\section{Exclusion criteria}

All patients meeting at least one of the following criteria were excluded: 1.) Patients whose liver or the right kidney was not clearly visualized on the same US image or ectopic right kidney or inadequate area of each organ to be sampled 2.) Patients whose renal echogenicity was altered by atrophy, numerous cortical cysts/masses, renal cortical scarring or hydronephrosis of the right kidney 3.) Patients with abnormal serum creatinine $>1.3 \mathrm{mg} / \mathrm{dL}^{(22)} 4$.) Patients with focal lesions in the right hepatic lobe causing distortion of liver echotexture or heterogeneous fat distribution in the liver.

\section{Ultrasonographic examination}

All participants underwent upper abdominal examination with ultrasonography in B-mode (GE logiq E9 with a 1-6 MHz Broad-spectrum convex transducer) by board-certified radiologists. Various image parameters were adjusted for each examination. All images were transmitted to a Picture Archiving and Communication System (PACS; Fujifilm Synapse, Tokyo, Japan).

\section{Ultrasound image analysis}

All ultrasound image analyses were performed by two independent operators, using a region of interest (ROI) tool on standard workstation from DICOM images on PACS without using extra-image analysis software.

The first operator was a radiologist with more than 8 years of experience in advance abdominal imaging, and the second operator was a radiology resident. Both of them were blinded to patients' clinical details. The hepatic and right renal ROIs were selected at the same depth along the focusing area near the center of the image to avoid image distortion effects and beam attenuation. An ROI in the liver parenchyma of $200-250 \mathrm{~mm}^{2}$ was selected, excluding blood vessels, bile ducts or focal liver lesions. An ROI of the right renal cortex of $20-30 \mathrm{~mm}^{2}$ was selected, excluding large vessels, renal sinus, masses or cysts. Portions of ROIs affected by artifacts were also excluded (Fig. 1).

The sonographic HR ratio was calculated by dividing the mean echo intensity of the pixels within the selected hepatic ROI by those within the selected ROIs of the right renal cortex. 


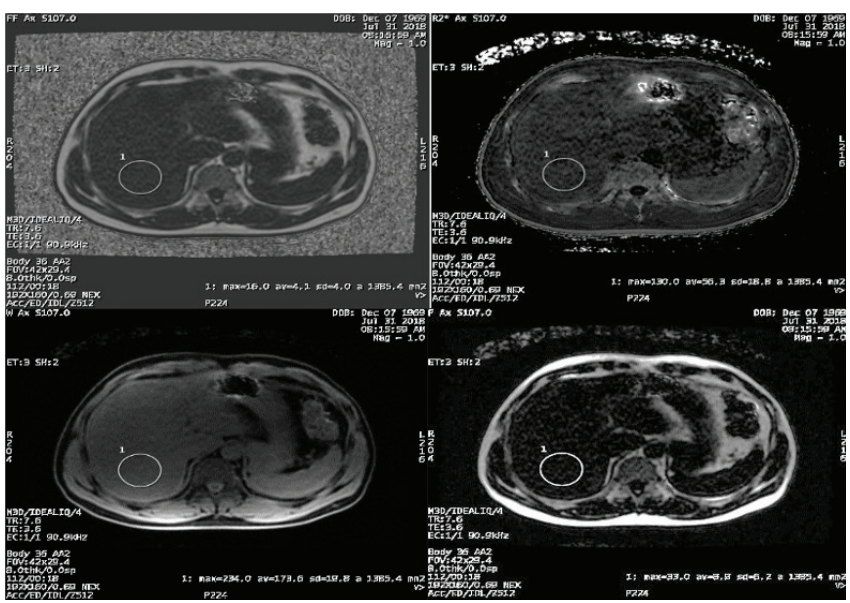

Fig. 2. The images were processed by using the software provided by the manufacturer (IDEAL-IQ; GE Healthcare) which created water, fat, $R 2 *$ maps and PDFF parametric map representing fat mapping of the entire liver. ROI was placed in hepatic segment VI

MRI examinations were performed using 3T GE Discovery MR750w. All participants underwent PDFF examination using complex-based, chemical shift-based, water and fat separation technique with low-flip angle, T1-weighted, 2D multiple-echo, spoiled gradient-echo sequence. The images were processed by using the software provided by the manufacturer (IDEAL-IQ; GE Healthcare) which created water, fat, R2* maps and PDFF parametric map representing fat mapping of the entire liver (Fig. 2). This technique provided correction of confounding factors on MR signal intensity, such as T1 bias, T2* decay, spectral complexity of fat, noise bias, and eddy currents ${ }^{(6)}$. (Detailed imaging parameters are as follows: TR7.6/TE3.6, flip angle $4^{\circ}$, bandwidth $90.91 \mathrm{~Hz} /$ pixel, number of excitations, 0.5; field of view $42 \mathrm{~cm}$; section thickness, $8 \mathrm{~mm}$; intersection gap, 0 , matrix $192 \times 160$. We acquired data sets with eight different echo times that ranged from 1.25 to $11.83 \mathrm{msec}$.)

MR elastography (MRE) was also performed in all individuals with $60-\mathrm{Hz}$ vibrations applied to the abdominal wall using a pneumatic actuator (MR-Touch; GE Healthcare), with two-dimensional gradient-echo MR elastography sequence and the direct inversion algorithm, previously described elsewhere ${ }^{(26)}$. (Detailed imaging parameters were as follows: TR/TE, 1000.3/63 ms; bandwidth $250 \mathrm{~Hz} /$ pixel; field-of-view (FOV), $38 \mathrm{~cm}$; matrix, $64 \times 64$; NEX, 2; slice thickness, $7.0 \mathrm{~mm}$ with a $2.5-\mathrm{mm}$ intersection gap.)

\section{PDFF measured by MRI image processing}

The radiology resident (the second operator), who was blinded to the patients' clinical details, manually performed circular ROI measurements in the hepatic segment VI on the PDFF parametric map to obtain fat fraction percentage using a work station provided by the manufacturer: AW4.4; GE healthcare. ROIs were selected to be $>400 \mathrm{~mm}^{2}$ avoiding major blood vessels, intrahepatic bile ducts, focal lesions and the lateral margin of the liver.

\section{Other data}

MRI imaging was reviewed using PACS; Fujifilm Synapse, Tokyo, Japan. Liver stiffness values $(\mathrm{kPa})$ were recorded. All MRE image analyses were performed by radiology residents and the quality was approved by advanced abdominal imaging radiologists.

Medical data records of each patient were obtained from hospital information system (HIS) and laboratory record. Baseline demographic, physical and biochemical data of the study participants included age, sex, weight, height, body mass index (BMI), alcohol use, hepatic viral profile, total bilirubin, albumin, SGPT, ALP, total cholesterol, low density lipoprotein (LDL) and triglycerides.

\section{Statistical analysis}

All statistical analyses were performed using Stata version 14.0. For descriptive analyses, categorical variables were reported in numbers and percentages.

Continuous variables were reported as means (SD) and ranges or medians and interquartile ranges (IQR). The Student's t-test and Mann-Whitney U-test were applied for the comparison of variables in different categories. Kruskal-Wallis $\mathrm{H}$ test was used for the assessment of differences in HR ratio between different grades of steato-

sis. After a positive Kruskal-Wallis H test ( $p$-value <0.05), a post-hoc analysis was used to perform pairwise comparisons between subgroups.

Spearman's correlation was used for the assessment of a correlation between continuous variables. The diagnostic performance of HR compared with MRI-PDFF was assessed by using receiver operating characteristic (ROC) curve. We determined the optimal HR ratio cutoff points for the diagnosis and grading of hepatic steatosis, as well as the sensitivity and specificity for each cutoff point. The HR ratios for statistical analysis were performed by the first operator who was more experienced in the field. The HR ratio interobserver agreement was determined using intraclass correlation coefficient (ICC) $(p$-values $<0.05$ were statistically significant and all tests were two tailed.)

\section{Reference standard for optimal threshold value of MRI-PDFF}

Currently, there are still no exact standardized fat fraction cutoff values for normal and abnormal liver fat.

We determined hepatic steatosis at $\mathrm{PDFF} \geq 5.56 \%$, which is a common threshold to distinguish normal from abnormal. This threshold was derived from a large MR spectroscopy study performed by Szczepaniak et al. in 2349 participants of the Dallas Heart Study ${ }^{(3)}$. Using this threshold, MRIPDFF accuracy was close to $100 \%$ for the detection of steatosis compared with MRS $^{(13)}$. 


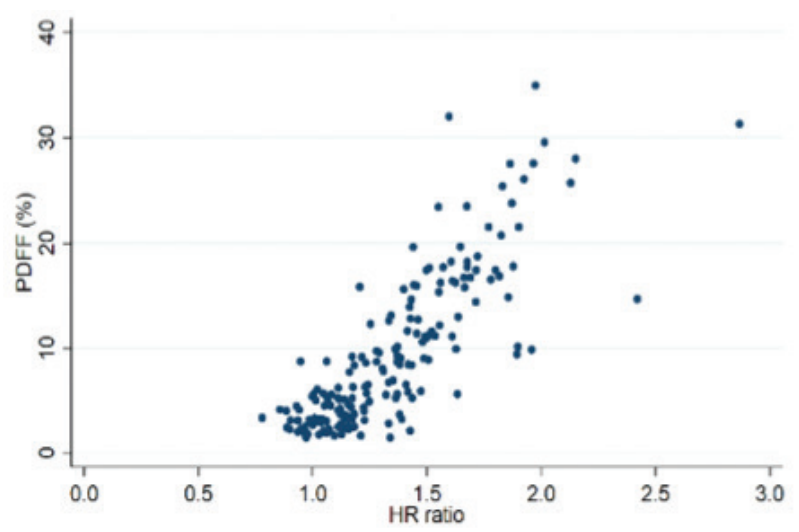

Spearman's correlation coefficient $=0.830, \mathrm{P}<0.001$

Fig. 3. Spearman's correlation between $H R$ ratio and fat fraction quantified by MRI-PDFF $3 T(N=179)$

We classified liver steatosis according to previous study by Middleton et al., as follows: Grade 2: $\mathrm{PDFF} \geq 16.3 \%$ and Grade 3: PDFF $\geq 21.7 \%$ (27).

According to the suggested guidelines for the interpretation of liver stiffness with MR elastography at $60 \mathrm{~Hz}^{(26)}$, we determined abnormal increased liver stiffness at $\geq 2.9$ kilopascals $(\mathrm{kPa})$ and performed staging of liver fibrosis as follows: normal or chronic inflammation: $<2.9 \mathrm{kPa}$, stage 1-2 fibrosis: 2.9-3.5 kPa, stage 2-3 fibrosis: $3.5-4.0 \mathrm{kPa}$, stage 3-4 fibrosis: $4.0-5.0 \mathrm{kPa}$ and stage 4 fibrosis/cirrhosis: $\geq 5.0 \mathrm{kPa}$.

\section{Results}

A total of179 patients were included in this study ( 82 males and 97 females; mean age of 53 years; range: $23-77$ years). The indications for abdominal MR imaging included nonalcoholic fatty liver disease $(n=97)$, hepatitis $C$ virus (HCV) infection $(n=59)$, psoriasis on methotrexate treatment $(n=4)$, HBV and HCV coinfection $(n=2)$, post liver transplantation with HCV infection $(n=2)$, post liver transplantation $(n=2)$, patients treated with chemotherapy $(n=1)$, unexplained elevation of liver enzymes $(n=1)$ and unknown etiology $(n=11)$.

Hepatic fat content by MRI-PDFF ranged from 1.5to $35.0 \%$. Based on the diagnostic criteria for steatosis, 75 patients had no steatosis $(75 / 179,42 \%)$ and 104 patients had steatosis $(104 / 179,58 \%)$. Grade $1(\geq 5.56 \%$ and $<16.3)$, grade $2(\geq 16.3 \%$ and $<21.7 \%)$ and grade $3(\geq 21.7 \%)$ steatosis was found in $71(40 \%), 20(11 \%)$ and $13(7 \%)$ patients, respectively. The median age of patients without steatosis was 58 years, and the median age of patients with steatosis was 51 years; the difference was statistically significant ( $p=0.007)$. BMI was statistically significantly higher in the steatosis group than non-steatosis group $(26.9 \pm 3.4$ vs. $23.7 \pm 3.5 \mathrm{~kg} / \mathrm{m}^{2} ; p$-value $\left.<0.001\right)$. The patients in the steatosis group had elevated triglyceride and SGPT levels compared with the non-steatosis group $(p<0.05)$. There was no significant difference in total cholesterol, low density lipoprotein (LDL), albumin, TB or ALP between these
Tab. 1. Demographic data of the 179 patients in the study sample

\begin{tabular}{|l|c|c|c|}
\hline \multicolumn{1}{|c|}{ Characteristics } & $\begin{array}{c}\text { Non-steatosis } \\
\text { group }(\boldsymbol{N}=\mathbf{7 5})\end{array}$ & $\begin{array}{c}\text { Steatosis group } \\
(\mathbf{N}=\mathbf{1 0 4})\end{array}$ & $\boldsymbol{p}$-value \\
\hline Age (y), median (IQR) & $58(49-64)$ & $51(41-60)$ & $\mathbf{0 . 0 0 7}$ \\
\hline BMI (kg/m²), mean (SD) & $23.7(3.5)$ & $26.9(3.4)$ & $<\mathbf{0 . 0 0 1}$ \\
\hline TC, mean (SD) & $189.9(41.0)$ & $201.8(40.6)$ & 0.111 \\
\hline TG, median (IQR) & $117(82-141)$ & $137(97-187)$ & $\mathbf{0 . 0 3 3}$ \\
\hline LDL (mg/dL), mean (SD) & $113.7(38.4)$ & $121.2(38.5)$ & 0.11 \\
\hline Albumin, median (IQR) & $4.1(3.8-4.3)$ & $4.2(4.0-4.4)$ & 0.059 \\
\hline TB, median (IQR) & $0.78(0.56-1.30)$ & $0.73(0.57-0.94)$ & 0.884 \\
\hline SGPT, median (IQR) & $32.5(19-55)$ & $45(29-64)$ & $\mathbf{0 . 0 3 3}$ \\
\hline ALP, median (IQR) & $79.0(61-101)$ & $77(61-88)$ & 0.557 \\
\hline HR ratio, median (IQR) & $1.11(1.01-1.18)$ & $1.49(1.34-1.68)$ & $<\mathbf{0 . 0 0 1}$ \\
\hline $\begin{array}{l}\text { Abbreviations: SD - standard deviation, IQR - interquartile range, LDL - } \\
\text { low-density lipoprotein , TG - serum trigleceride, BMI - body mass } \\
\text { index (kg/m²), SGPT - serum glutamate-pyruvate transaminase, ALP - } \\
\text { alkaline phosphatase, HR ratio - hepatorenal ratio }\end{array}$ \\
\hline
\end{tabular}

Tab. 2. Correlation between hepatorenal ratio and MRI-PDFF by liver stiffness $(n=179)$

\begin{tabular}{|c|c|c|c|}
\hline $\begin{array}{c}\text { Liver stiffness by MRE } \\
(\mathbf{k P a})\end{array}$ & $\mathbf{N}$ & $\begin{array}{c}\text { Spearman's } \\
\text { correlation coefficient }\end{array}$ & $\boldsymbol{p}$-value \\
\hline$<2.5$ & 87 & 0.874 & $<0.001$ \\
\hline $2.5-2.8$ & 18 & 0.849 & $<0.001$ \\
\hline $2.9-3.4$ & 26 & 0.832 & $<0.001$ \\
\hline$\geq 3.5-5$ & 48 & 0.591 & $<0.001$ \\
\hline
\end{tabular}

two groups. Demographic data of the 179 patients in the study sample are summarized in Tab. 1.

The HR ratio was significantly highly correlated with MRI-PDFF (Spearman's correlation coefficient $=0.83$ ) $(p<0.001)$ (Fig. 3). In subgroup analysis, there was a higher correlation between HR ratio and MRI-PDFF in patients with normal liver stiffness $(n=105)$ than those with increased liver stiffness $(n=74)$ (Spearman's correlation coefficient $=0.87$ vs. 0.69 , respectively, $p=0.0017$ ). High correlation of HR ratio with MRI-PDFF was observed

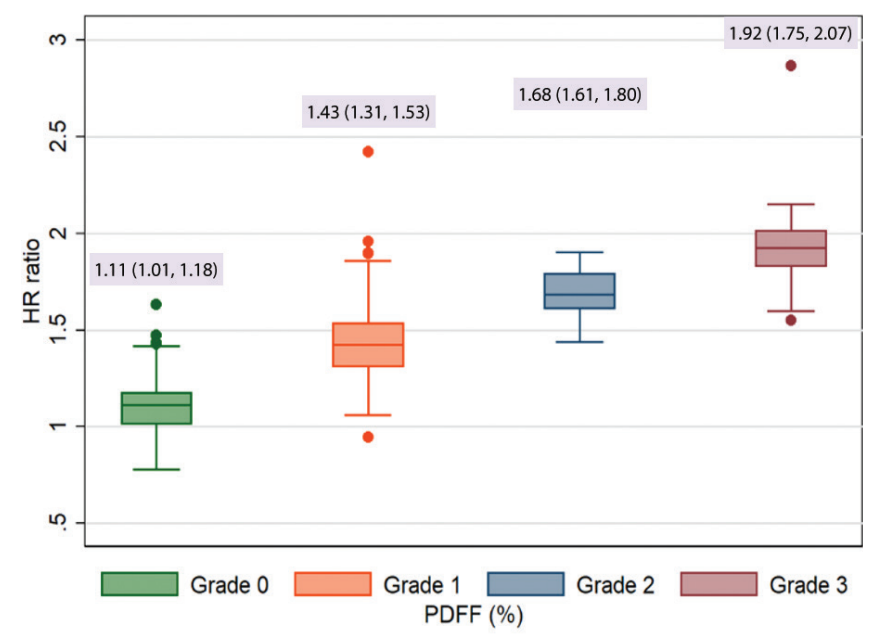

Fig. 4. Distribution of hepatorenal ratio in patients according to categories of steatosis; none, grade 1, grade 2 and grade 3 steatosis 


\begin{tabular}{|l|l|l|l|}
\hline \multicolumn{3}{|c|}{ All patients, N=179 } \\
\hline ROC & \multicolumn{2}{|c|}{ PDFF $\geq \mathbf{5 . 5 6 \%}$} & \multicolumn{2}{c|}{ PDFF $\geq \mathbf{1 6 . 3 \%}$} \\
\hline
\end{tabular}

Fig. 5. ROC curve of sensitivity (true-positive fraction) plotted against 1-specificity (false-positive fraction) of hepatorenal ratio for the diagnosis of different levels of steatosis quantified by MRI-PDFF

in patients with less than stage 2 fibrosis, whereas moderate correlation of HR ratio with MRI-PDFF was found in patients with stage 2 fibrosis or higher (Tab. 2).

The median of HR ratio was 1.31 (1.10-1.55), and the range was $0.78-2.87$. There was a significant difference in the median of HR ratio between patients with steatosis and those without, i.e. $1.49(1.34-1.68)$ vs. $1.11(1.01-1.18)$ $(p<0.001)$, respectively. Higher HR ratio corresponded with higher PDFF. There were also significant differences in the median HR ratio among sub-groups $(p<0.001)$ except for between grade 2 and 3 steatosis, which was marginally significant ( $p=0.05$ ) (Fig. 4).

\section{ROC analysis and the HR ratio cutoff points}

The appropriate HR ratio cutoff points were selected on the basis of the sensitivity and specificity, using the ROC analysis. The optimal cutoff for the HR ratio to define steatosis

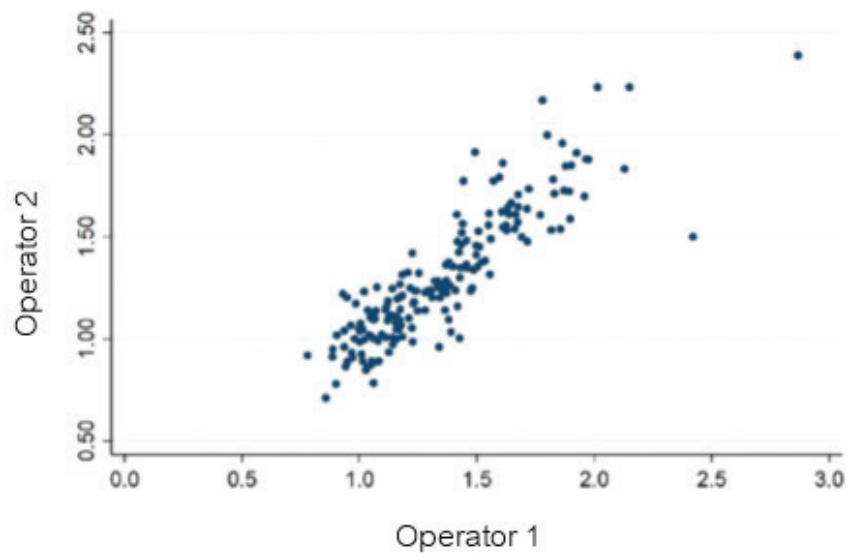

Fig. 6. Hepatorenal ratio interobserver reliability. Intraclass correlation coefficient $=0.882, \mathrm{p}<0.001$. Spearman's correlation coefficient $=0.889, \mathrm{p}<0.001$ at MRI-PFFF $\geq 5.56 \%$ was a value equal to or greater than 1.18 , with a sensitivity of $90.0 \%$ (95\% CI, 83.0-95.3\%), specificity of $80.0 \%$ (95\% CI, 69.2-88.4\%) and AUC 92.6 (95\% CI, 88.8- 96.4\%), PPV 86.2\% (95\% CI, 79.9-90.8\%) and NPV $85.7 \%$ (95\% CI, 76.7-91.6\%).

The optimal HR ratio cutoff point for prediction of grade 2 hepatic steatosis $\geq 16.3 \%$ was 1.55 , with sensitivity of $90.9 \%(95 \% \mathrm{CI}, 75.7-98.1 \%)$ and specificity of $89.7 \%$ (95\% CI, 83.6- 94.1\%), AUC 95\% (95\% CI, 92.4-98.2\%), PPV $66.7 \%$ (95\% CI 55.0-76.6\%) and NPV 97.7\% (95\% CI, 93.7-99.2\%). The optimal HR ratio cutoff point for the prediction of grade 3 hepatic steatosis $\geq 21.7 \%$ was 1.60 , with sensitivity of $92.3 \%$ (95\% CI, 64.0-99.8\%) and specificity of $83.1 \%$ (95\% CI, 76.6-88.5\%), AUC 95\% (95\% CI, 91.1$99.2 \%$ ), PPV $30.0 \%$ (95\% CI, $22.8-38.4 \%$ ), NPV $99.3 \%$ (95\% CI, 95.5-99.9\%) (Fig. 5).

\section{Interobserver reliability}

The reproducibility of HR ratios was assessed by intraclass correlation coefficient (ICC), showing a high correlation between two independent operators (ICC $=0.882$, $p<0.001$ ) (Fig. 6).

\section{Discussion}

Nowadays, ultrasonography is usually the most initial modality to assess hepatic steatosis. The diagnosis of hepatic steatosis on ultrasonography involves subjective evaluation of increased liver echogenicity, portal vein blurring, beam attenuation and poor visualization of diaphragm. Prior studies found that abnormal hepatorenal echogenicity was the most sensitive among other sonographic signs in the diagnosis of hepatic steatosis ${ }^{(18,28)}$. Sonographic parameters for quantitative assessment of hepatic steatosis, 
including HR ratio, have been previously investigated. Prior studies have reported high correlation between sonographic HR ratio and histologic steatosis among patients with various liver diseases $(r=0.71-0.82)^{(20,22)}$ and also in patients with NAFLD $(r=0.80)^{(23)}$. There was also a high correlation between sonographic HR ratio and ${ }^{1} \mathrm{H}-\mathrm{MRS}$ in patients with NAFLD $(\mathrm{r}=0.89-0.95)^{(24,25)}$. Therefore, HR ratio may offer a potentially useful quantitative tool for hepatic steatosis.

The previously reported HR cutoff ratios are varied, depending on the characteristics of the target population, standard reference and the selected threshold. For example, Webb et al. ${ }^{(20)}$ and Marshall et al. ${ }^{(22)}$ reported HR ratio cutoff value using specialized software at 1.49 and 1.28 , respectively, for identifying more than $5 \%$ of fat from biopsy in patients with various liver diseases. Shiralkar ${ }^{(29)}$ investigated the same patients included in the study by Marshall et al., and found that the HR ratio can be accurately calculated from PACS without specialized software yielding a cutoff point at 1.34. Borges et al. ${ }^{(23)}$ reported a cutoff ratio at 1.24 in patients with only NAFLD using the same threshold from biopsy. Mancini et al. ${ }^{(22)}$ and Martin-Rodriguez ${ }^{(24)}$ investigated HR ratio in patients with NAFLD for the prediction of ${ }^{1} \mathrm{H}$-MRS of at least $5 \%$, yielding the best HR ratio cutoff point at 2.2 and 1.28 , respectively. However, there is no perfect agreement in the percentage of fat from liver biopsy and MR techniques since they measure different aspects of hepatic steatosis. Also, a direct comparison between sonographic HR ratio and MRI-PDFF has not been performed yet.

In this study, we assessed sonographic HR ratio to predict the degree of fat content measured by MRI-PDFF in patients with liver disease of varying etiology. We calculated HR ratio using standard workstation without additional software because we attempted to keep the method simple so as to be easily applied and repeated. In this study, we measured PDFF on parametric maps at hepatic segment VI because the sonographic HR ratios were calculated in this segment.

Our study revealed a high correlation between sonographic HR ratio and percentage fat fraction measured by MRI-PDFF. We found that HR ratio could provide diagnosis of small fat content as low as PDFF of $5.56 \%$ with good accuracy. In our population, the HR cutoff ratio for predicting hepatic steatosis at 1.18 yielded sensitivity of $90.0 \%$ and specificity of $80.0 \%$. Our study shared similar

findings with the study by Kim et al. ${ }^{(28)}$ which correlated

conventional ultrasonography signs with the MRI-PDFF. They found that PDFF was $2.6 \%$ (S.D. \pm 1.22 ) in subjects without any signs of hepatic steatosis, while PDFF was $5.4 \%$ (S.D. \pm 3.35 ) when abnormal hepatorenal echoes were present. Abnormal hepatorenal echoes yielded 96.6\% sensitivity, $74.8 \%$ specificity and $87.5 \%$ AUC for detecting $6.4 \%$ PDFF.

Interference of liver fibrosis in liver parenchymal echogenicity had been previously investigated. Some studies ${ }^{(18,30)}$ showed that bright liver echogenicity significantly correlated with steatosis, but not fibrosis. Borges et al. ${ }^{(23)}$ also reported that HR ratio was more strongly correlated with steatosis than fibrosis on liver biopsy. On the other hand, Perez et al. showed that US was inaccurate for diagnosing hepatic steatosis in patients with chronic liver disease due to the higher probability of hepatic fibrosis or inflammation in these patients, causing echogenic alteration seen in US ${ }^{(31)}$. Stiffness value measured by MRE is now the most accurate noninvasive method for the detection of liver fibrosis ${ }^{(26)}$. In our study, we found a reduced correlation between HR ratio and MRI-PDFF in patients with $\geq$ stage 2 fibrosis. Therefore, the HR ratio should be used carefully in this group of patients.

Nowadays, liver fibrosis can be assessed not only with MRE, but also with ultrasound elastography (UE), providing comprehensive one-time assessment of liver steatosis and fibrosis. However, confounding of steatosis grade on the fibrosis estimation of UE is controversial and still under investigated ${ }^{(32,33)}$.

Limitations of this study include its retrospective design. Also, this study had no histologic evaluation, which is the gold standard of quantification of hepatic steatosis. Furthermore, the result may be affected by selection bias since the study population who underwent MRI and US at our institution were not representative of the general population. Also, sonographic HR ratio could be assessed only in patients who had adequate sample area of each organ on ultrasonographic image and without focal lesions or diseases of the liver and the right kidney, which may alter their echogenicity. Due to the nature of heterogeneous distribution of hepatic steatosis, it might lead to sampling errors, which can occur whatever method is used. The population in our study was heterogeneous due to the broad etiologies of liver disease that can be responsible for liver steatosis. However, we believe that the population might be more representative of the general population rather than focused on patients with NAFLD only.

Further validation is needed, including future study in larger populations and evaluation of HR ratio in the detection of subtle change of fat content for follow-up study.

\section{Conclusion}

In conclusion, the sonographic HR ratio was highly correlated with PDFF measured by MRI, easily applicable using standard workstation without additional software, and also reproducible. It could become an effective tool for quantitative evaluation of hepatic steatosis alternative to MRI-PDFF.

\section{Conflict of interest}

Authors do not report any financial or personal connections with other persons or organizations, which might negatively affect the contents of this publication and/or claim authorship rights to this publication. 


\section{References}

1. Reeder SB, Sirlin CB: Quantification of liver fat with magnetic resonance imaging. Magn Reson Imaging Clin N Am 2010; 18: 337-357, ix.

2. Abd El-Kader SM, El-Den Ashmawy EM: Non-alcoholic fatty liver disease: The diagnosis and management. World J Hepatol 2015; 7: 846-858.

3. Szczepaniak LS, Nurenberg P, Leonard D, Browning JD, Reingold JS, Grundy S et al:: Magnetic resonance spectroscopy to measure hepatic triglyceride content: prevalence of hepatic steatosis in the general population. Am J Physiol Endocrinol Metab 2005; 288: E462-E468.

4. Lok AS, Everhart JE, Chung RT, Padmanabhan L, Greenson JK, Shiffman ML et al.: Hepatic steatosis in hepatitis C: comparison of diabetic and nondiabetic patients in the hepatitis $\mathrm{C}$ antiviral longterm treatment against cirrhosis trial. Clin Gastroenterol Hepatol 2007; 5: 245-254.

5. Fartoux L, Chazouilleres O, Wendum D, Poupon R, Serfaty L: Impact of steatosis on progression of fibrosis in patients with mild hepatitis C. Hepatology 2005; 41: 82-87.

6. Reeder SB, Cruite I, Hamilton G, Sirlin CB: Quantitative assessment of liver fat with magnetic resonance imaging and spectroscopy. J Magn Reson Imaging 2011; 34: 729-749.

7. National Guideline Centre (UK): Non-alcoholic fatty liver disease: assessment and management. London: National Institute for Health and Care Excellence (UK) 2016; 6. Dostęp online: https://www.ncbi.nlm.nih.gov/ books/NBK384715/.

8. Regev A, Berho M, Jeffers LJ, Milikowski C, Molina EG, Pyrsopoulos NT et al.: Sampling error and intraobserver variation in liver biopsy in patients with chronic HCV infection. Am J Gastroenterol 2002; 97: 2614-2618.

9. Ratziu V, Charlotte F, Heurtier A, Gombert S, Giral P, Bruckert E et al.: Sampling variability of liver biopsy in nonalcoholic fatty liver disease. Gastroenterology 2005; 128: 1898-1906.

10. Kinner S, Reeder SB, Yokoo T: Quantitative Imaging Biomarkers of NAFLD. Dig Dis Sci 2016; 61: 1337-1347.

11. Noureddin M, Lam J, Peterson MR, Middleton M, Hamilton G, Le TA et al.: Utility of magnetic resonance imaging versus histology for quantifying changes in liver fat in nonalcoholic fatty liver disease trials. Hepatology 2013; 58: 1930-1940.

12. Kang GH, Cruite I, Shiehmorteza M, Wolfson T, Gamst AC, Hamilton G et al.: Reproducibility of MRI-determined proton density fat fraction across two different MR scanner platforms. J Magn Reson Imaging 2011; 34: 928-934.

13. Meisamy S, Hines CD, Hamilton G, Sirlin CB, McKenzie CA, Yu H et al.: Quantification of hepatic steatosis with T1-independent, T2-corrected MR imaging with spectral modeling of fat: blinded comparison with MR spectroscopy. Radiology 2011; 258: 767-775.

14. Idilman IS, Aniktar H, Idilman R, Kabacam G, Savas B, Elhan A et al.: Hepatic steatosis: quantification by proton density fat fraction with MR imaging versus liver biopsy. Radiology 2013; 267: 767-775.

15. Rehm JL, Wolfgram PM, Hernando D, Eickhoff JC, Allen DB, Reeder SB: Proton density fat-fraction is an accurate biomarker of hepatic steatosis in adolescent girls and young women. Eur Radiol 2015; 25: 2921-2930.

16. Permutt Z, Le TA, Peterson MR, Seki E, Brenner DA, Sirlin C et al.: Correlation between liver histology and novel magnetic resonance imaging in adult patients with non-alcoholic fatty liver disease - MRI accurately quantifies hepatic steatosis in NAFLD. Aliment Pharmacol Ther 2012; 36: 22-29.

17. Tang A, Tan J, Sun M, Hamilton G, Bydder M, Wolfson T et al.: Nonalcoholic fatty liver disease: MR imaging of liver proton density fat fraction to assess hepatic steatosis. Radiology 2013; 267: 422-431.
18. Palmentieri B, de Sio I, La Mura V, Masarone M, Vecchione R, Bruno S et al.: The role of bright liver echo pattern on ultrasound B-mode examination in the diagnosis of liver steatosis. Dig Liver Dis 2006; 38: 485-489.

19. Osawa H, Mori Y: Sonographic diagnosis of fatty liver using a histogram technique that compares liver and renal cortical echo amplitudes. J Clin Ultrasound 1996; 24: 25-29.

20. Webb M, Yeshua H, Zelber-Sagi S, Santo E, Brazowski E, Halpern Z et al.: Diagnostic value of a computerized hepatorenal index for sonographic quantification of liver steatosis. AJR Am J Roentgenol 2009; 192 : 909-914.

21. Mancini M, Prinster A, Annuzzi G, Liuzzi R, Giacco R, Medagli C et al.: Sonographic hepatic-renal ratio as indicator of hepatic steatosis: comparison with ${ }^{1} \mathrm{H}$ magnetic resonance spectroscopy. Metabolism 2009; 58 : 1724-1730.

22. Marshall RH, Eissa M, Bluth EI, Gulotta PM, Davis NK: Hepatorenal index as an accurate, simple, and effective tool in screening for steatosis. AJR Am J Roentgenol 2012; 199: 997-1002.

23. Borges VF, Diniz AL, Cotrim HP, Rocha HL, Andrade NB: Sonographic hepatorenal ratio: a noninvasive method to diagnose nonalcoholic steatosis. J Clin Ultrasound 2013; 41: 18-25.

24. Martin-Rodriguez JL, Arrebola JP, Jimenez-Moleon JJ, Olea N, GonzalezCalvin JL: Sonographic quantification of a hepato-renal index for the assessment of hepatic steatosis in comparison with 3T proton magnetic resonance spectroscopy. Eur J Gastroenterol Hepatol 2014; 26: 88-94.

25. Zhang B, Ding F, Chen T, Xia LH, Qian J, Lv GY: Ultrasound hepatic/renal ratio and hepatic attenuation rate for quantifying liver fat content. World J Gastroenterol 2014; 20: 17985-17992.

26. Babu AS, Wells ML, Teytelboym OM, Mackey JE, Miller FH, Yeh BM et al.: Elastography in chronic liver disease: modalities, techniques, limitations, and future directions. Radiographics 2016; 36: 1987-2006.

27. Middleton MS, Heba ER, Hooker CA, Bashir MR, Fowler KJ, Sandrasegaran $\mathrm{K}$ et al.: Agreement between magnetic resonance imaging proton density fat fraction measurements and pathologist-assigned steatosis grades of liver biopsies from adults with nonalcoholic steatohepatitis. Gastroenterology 2017; 153: 753-761.

28. Kim M, Kang B-K, Jun DW: Comparison of conventional sonographic signs and magnetic resonance imaging proton density fat fraction for assessment of hepatic steatosis. Sci Rep 2018; 8: 7759.

29. Shiralkar K, Johnson S, Bluth EI, Marshall RH, Dornelles A, Gulotta PM: Improved method for calculating hepatic steatosis using the hepatorenal index. J Ultrasound Med 2015; 34: 1051-1059.

30. Loy M, Serra G, Chessa L: The prevalence of bright liver echo pattern in patients with chronic hepatitis $\mathrm{C}$ : correlation with steatosis and fibrosis. J Ultrasound 2014; 19: 91-98.

31. Perez NE, Siddiqui FA, Mutchnick MG, Dhar R, Tobi M, Ullah N et al:: Ultrasound diagnosis of fatty liver in patients with chronic liver disease: a retrospective observational study. J Clin Gastroenterol 2007; 41: 624629.

32. Petta S, Maida M, Macaluso FS, Di Marco V, Cammà C, Cabibi D, Craxì A: The severity of steatosis influences liver stiffness measurement in patients with nonalcoholic fatty liver disease. Hepatology 2015; 62: 1101-1110.

33. Samir AE, Dhyani M, Vij A, Bhan AK, Halpern EF, Méndez-Navarro J et al.: Shear-wave elastography for the estimation of liver fibrosis in chronic liver disease: determining accuracy and ideal site for measurement. Radiology 2015; 274: 888-896. 